\title{
Energy Levels of Singly-Ionized Platinum
}

\section{Jean Blaise and Jean-François Wyart}

Laboratoire Aimé Cotton, ${ }^{1}$ Bât. 505, C.N.R.S. II, Centre Universitaire, F-91405-ORSAY (France)
The analysis of $\mathrm{Pt}$ II is extended by using accurate wavelength measurements by Sansonetti et al. Forty-three new even and 104 new odd levels have been found. The Slater-Condon parametric method is used for the interpretation of the $5 d^{9}, 5 d^{8} 6 s$, and $5 d^{7} 6 s^{2}$ low even configurations and the $5 d^{8}(7 s+6 d)$ high even configurations with root mean square deviations smaller than $80 \mathrm{~cm}^{-1}$.
The importance of the $5 d^{8}-5 d^{7} 6 s$ core interaction in interpreting the even-parity levels is stressed.

Key words: atomic spectroscopy; electronic configurations; energy levels; platinum.

Accepted: November 21, 1991

\section{Introduction}

The spectrum of platinum emitted by a hollow cathode lamp has been recently observed and measured [1]. The improved wavelengths of the classified lines led Reader et al. [2] to determine accurate energies for the known levels. The extensive line list comprised many unclassified lines. Their interpretation has been undertaken at Laboratoire Aimé Cotton in order to improve the knowledge of excited levels at the end of the $5 d$ period.

The strong unclassified lines have been interpreted in the present work with the support of theoretical energy level predictions and a computer program to search for recurring energy differences in the list of observed wave numbers. The measured wave numbers of classified lines deviate from the differences between their initial and final levels by less than $0.050 \mathrm{~cm}^{-1}$ if the lines are not blended with other transitions. The energy levels are reported in Tables 2, 3, and 5, in which the 3-digit

\footnotetext{
${ }^{1}$ In association with Université Paris-Sud.
}

values are taken from Ref. [2]. The $J$-values of some levels have been changed and the newly classified lines led to slight modifications of their energies. The uncertainty of the levels depends on the intensities and spectral regions of their transitions. It ranges from 0.050 to $0.100 \mathrm{~cm}^{-1}$. The classified lines are reported in Ref. [1].

\section{Interpretation of the Low Even-Parity Configurations $5 d^{9}, 5 d^{8} 6 s$, and $5 d^{7} 6 s^{2}$}

In 1977 [3], a systematic description of the even configurations $(5 d+6 s)^{N}$ was performed in the framework of the Slater-Condon parametric method. It was shown that configuration mixing was very important within these groups and led to the revision and limited extension of some analyses. In the absence of definite configuration assignments for many levels, the sum of the squared amplitudes represented $53 \%$ of all $5 d^{N}$ levels from Lu II to Au II, $56 \%$ for $5 d^{N-1} 6 s$ and only $27 \%$ for 
$5 d^{N-2} 6 s^{2}$. In Pt II, all 21 levels found by Shenstone [4] were supported by the theoretical calculation, but six of his empirical $L S$ designations did not correspond to the leading component of the eigenfunction. The $5 d^{7} 6 s^{2}$.configuration was limited to four known levels and the relevant energy parameters needed to be fixed or constrained. The present analysis was guided by the results of [3] and the number of levels of the $(5 d+6 s)^{9}$ group has been brought from 21 to 33 . The present interpretation of the three configurations $5 d^{9}, 5 d^{8} 6 s$ and $5 d^{7} 6 s^{2}$ leads to improved parameter values, a number of constraints in the least-squares fitting process being now removed. The present set of parameters includes: a constant energy for all three configurations, $A$, the energy differences between configurations, $S\left(d^{9}-d^{7} s^{2}\right)$ and $S\left(d^{8} s-d^{7} s^{2}\right)$, all Slater integrals describing the electrostatic interactions within the studied group, the effective electrostatic parameters $\alpha_{0}$ and $\beta_{0}$ as defined in the formalism of orthogonal operators [5], and finally, the usual spin-orbit parameters. These 18 parameters have been reduced to 13 adjustable ones by means of constraints detailed in Table 1 . These constraints were derived from earlier studies of $(5 d+6 s)^{N}$ groups. The root mean square deviation is $73 \mathrm{~cm}^{-1}$. The comparison of experimental and theoretical energies is given in Table 2. The theoretical data are limited to the theoretical energy $E_{\text {th }}$, the first component of the eigenfunction and the percentage of the components of the 3 configurations (squared amplitudes) in the eigenfunctions. The coefficients of the interaction parameter $R^{2}\left(5 d^{2}, 5 d 6 s\right)$ in intermediate coupling show that $5 d^{8} 6 s^{2} \mathrm{P}_{1 / 2}$ and $5 d^{7} 6 s^{2}{ }^{2} \mathrm{P}_{1 / 2}$ which are distant by $33700 \mathrm{~cm}^{-1}$ have a mutual repulsion of $7000 \mathrm{~cm}^{-1}$ and that four other levels of $5 d^{8} 6 s$ are shifted to lower energies by more than $2000 \mathrm{~cm}^{-1}$.

Table 1. Fitted energy parameters $\left(\mathrm{cm}^{-1}\right)$ of the even configurations of Pt II. Standard deviations of the parameters are given in parentheses

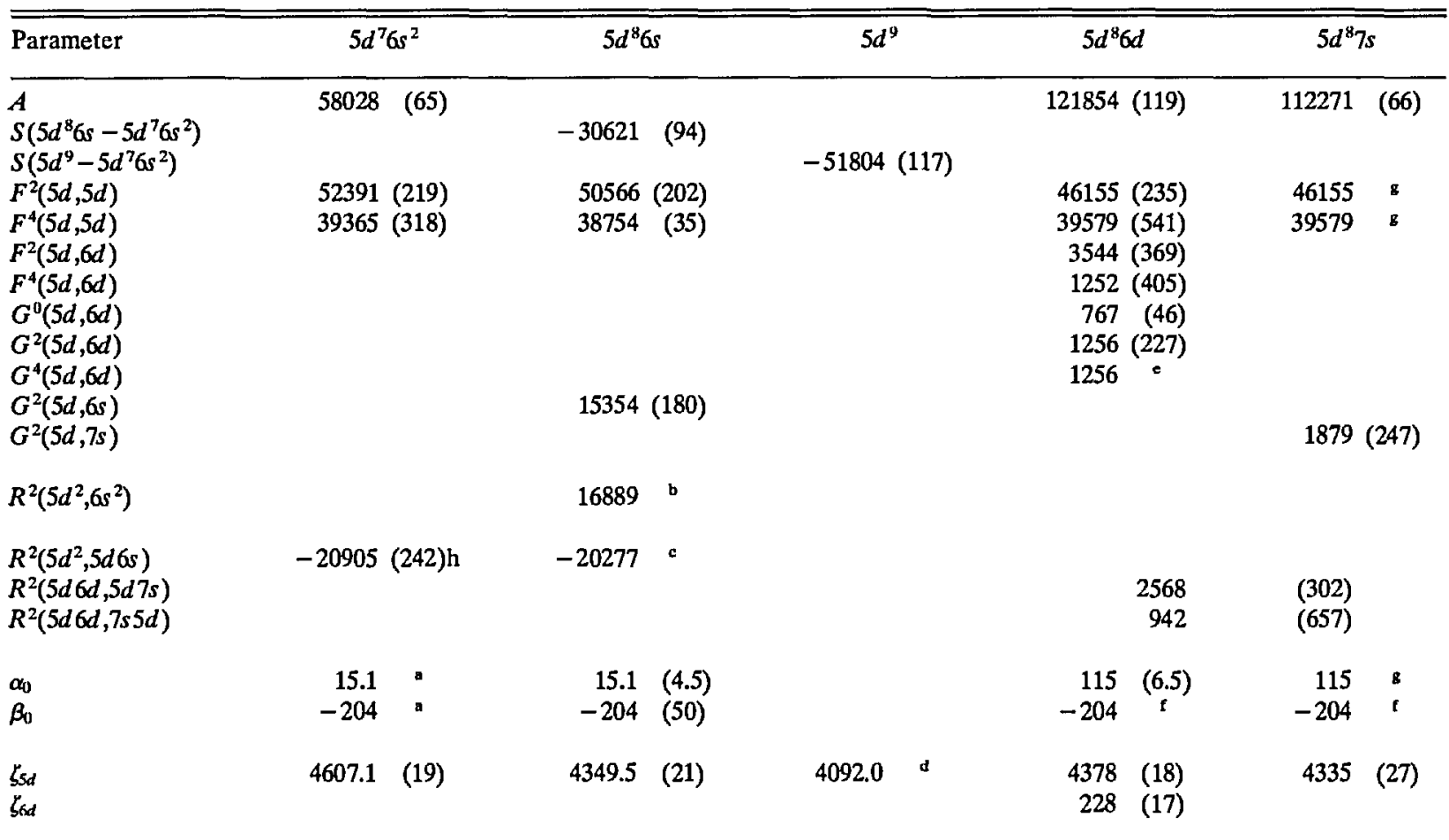

Parameters constrained to be equal in $5 d^{7} 6 s^{2}$ and $5 d^{8} 6 s$.

b The parameter $R^{2}\left(5 d^{2}, 6 s^{2}\right)$ of the $5 d^{7} 6 s^{2}-5 d^{9}$ interaction is held in a constant ratio with the $G^{2}(5 d, 6 s)$ of $5 d^{8} 6 s$.

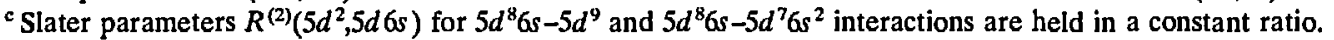

d $\zeta\left(5 d^{7} 6 s^{2}\right)+\zeta\left(5 d^{9}\right)=2 \zeta\left(5 d^{8} 6 s\right)$.

- $G^{2}(5 d, 6 d)=G^{4}(5 d, 6 d)$.

${ }^{t}$ Held fixed to the fitted value of the lowest configurations.

${ }^{8}$ Held equal to the same parameter in $5 d^{8} 6 d$.

h Parameter for the $5 d^{7} 6 s^{2}-5 d^{8} 6 s$ interaction. 
Table 2. Low even energy levels of Pt II. The theoretical energies $E_{\text {th }}$ are those of the mixed configurations $5 d^{9}, 5 d^{8} 6 s$ and $5 d^{7} 6 s^{2}$ (designated $\mathrm{A}, \mathrm{B}$, and $\mathrm{C}$ in the first components of the eigenfunction)

\begin{tabular}{|c|c|c|c|c|c|c|c|}
\hline \multirow{2}{*}{$\begin{aligned} \begin{array}{c}E_{\exp } \\
\left(\mathrm{cm}^{-1}\right)\end{array} & 0 \\
& 0\end{aligned}$} & \multirow{2}{*}{$\begin{array}{l}J \\
5 / 2\end{array}$} & \multirow{2}{*}{$\frac{\begin{array}{c}E_{\mathrm{th}} \\
\left(\mathrm{cm}^{-1}\right)\end{array}}{16}$} & \multicolumn{2}{|c|}{$\begin{array}{c}\text { First comp. } \\
\%\end{array}$} & \multirow{2}{*}{$\begin{array}{c}5 d^{9} \\
\% \\
90.7\end{array}$} & \multirow{2}{*}{$\begin{array}{c}5 d^{8} 6 s \\
\% \\
7.6\end{array}$} & \multirow{2}{*}{$\begin{array}{c}5 d^{7} 6 s^{2} \\
\% \\
1.7\end{array}$} \\
\hline & & & $A^{2} D$ & 90.7 & & & \\
\hline 4786.611 & $9 / 2$ & 4862 & $\mathrm{~B}^{4} \mathrm{~F}$ & 96.6 & 0 & 100. & 0 \\
\hline 8419.822 & $3 / 2$ & 8475 & $A^{2} D$ & 62.9 & 62.9 & 34.5 & 2.6 \\
\hline 9356.274 & $7 / 2$ & 9234 & $B^{4} \mathrm{~F}$ & 67.5 & 0 & 99.6 & 0.4 \\
\hline 13329.227 & $5 / 2$ & 13345 & $B^{4} \mathbf{P}$ & 36.2 & 5.5 & 94.1 & 0.4 \\
\hline 15791.276 & $3 / 2$ & 15639 & $A^{2} D$ & 32.3 & 32.3 & 65.1 & 2.6 \\
\hline 16820.894 & $5 / 2$ & 16770 & $\mathrm{~B}^{4} \mathrm{~F}$ & 60.0 & 0.4 & 99.3 & 0.3 \\
\hline 18097.715 & $7 / 2$ & 18171 & $\mathrm{~B}^{2} \mathrm{~F}$ & 63.8 & 0 & 98.8 & 1.2 \\
\hline 21168.684 & $3 / 2$ & 21146 & $\mathrm{~B}^{4} \mathrm{~F}$ & 39.5 & 0.1 & 94.1 & 5.8 \\
\hline 21717.260 & $1 / 2$ & 21774 & $\mathrm{~B}^{4} \mathrm{P}$ & 87.4 & 0 & 99.8 & 0.2 \\
\hline 23461.503 & $5 / 2$ & 23542 & $B^{2} F$ & 48.3 & 1.2 & 95.8 & 3.0 \\
\hline 23875.553 & $3 / 2$ & 23886 & $\mathrm{~B}^{4} \mathrm{P}$ & 56.3 & 0.3 & 87.4 & 12.3 \\
\hline 24879.480 & $9 / 2$ & 24846 & $\mathrm{C}^{4} \mathrm{~F}$ & 67.7 & 0 & 13.6 & 86.4 \\
\hline 27255.687 & $1 / 2$ & 27207 & $B^{2} \mathbf{P}$ & 77.8 & 0 & 84.7 & 15.3 \\
\hline 29030.479 & $7 / 2$ & 28968 & $B^{2} G$ & 88.0 & 0 & 94.5 & 5.5 \\
\hline 29261.967 & $9 / 2$ & 29341 & $B^{2} \mathbf{G}$ & 78.6 & 0 & 81.3 & 18.7 \\
\hline 32237.007 & $3 / 2$ & 32182 & $\mathrm{~B}^{2} \mathrm{D}$ & 53.3 & 3.4 & 85.6 & 11.0 \\
\hline 32918.561 & $5 / 2$ & 32981 & $\mathrm{~B}^{2} \mathrm{D}$ & 36.2 & 1.4 & 87.3 & 11.3 \\
\hline 34647.221 & $7 / 2$ & 34624 & $\mathrm{C}^{4} \mathrm{~F}$ & 95.2 & 0 & 1.5 & 98.5 \\
\hline 36484.028 & $5 / 2$ & 36555 & $\mathrm{C}^{4} \mathrm{~F}$ & 55.6 & 0.1 & 9.4 & 90.5 \\
\hline 37877.792 & $3 / 2$ & 37895 & $\mathrm{C}^{4} \mathrm{~F}$ & 43.7 & 0.1 & 12.9 & 87.0 \\
\hline N 41434.11 & $5 / 2$ & 41433 & $\mathrm{C}^{4} \mathrm{P}$ & 76.1 & 0.1 & 0 & 99.9 \\
\hline N 42031.85 & $3 / 2$ & 41986 & $\mathrm{C}^{4} \mathrm{P}$ & 50.1 & 0 & 11.9 & 88.1 \\
\hline N 43737.40 & $9 / 2$ & 43774 & $C^{2} G$ & 52.7 & 0 & 4.5 & 95.5 \\
\hline N 46046.43 & $1 / 2$ & 46086 & $\mathrm{C}^{4} \mathrm{P}$ & 76.6 & 0 & 9.0 & 91.0 \\
\hline N 48591.04 & $11 / 2$ & 48524 & $\mathrm{C}^{2} \mathrm{H}$ & 100. & 0 & 0 & 100. \\
\hline \multirow[t]{2}{*}{ N 50564.60} & $7 / 2$ & 50607 & $C^{2} G$ & 79.8 & 0 & 4.2 & 95.8 \\
\hline & $1 / 2$ & 53204 & $B^{2} S$ & 76.6 & 0 & 86.6 & 13.4 \\
\hline N 53749.63 & $3 / 2$ & 53722 & $\mathrm{C}^{4} \mathrm{P}$ & 50.1 & 0 & 3.8 & 96.2 \\
\hline N 54373.47 & $5 / 2$ & 54333 & $\mathrm{C}_{3}^{2} \mathrm{D}$ & 53.5 & 0.1 & 2.5 & 97.4 \\
\hline N 58062.04 & $5 / 2$ & 58072 & $\mathrm{C}^{2} \mathrm{~F}$ & 81.7 & 0.1 & 3.9 & 96.1 \\
\hline N 58491.21 & $9 / 2$ & 58518 & $\mathrm{C}^{2} \mathrm{H}$ & 68.1 & 0 & 0.6 & 99.4 \\
\hline N 60986.75 & $1 / 2$ & 60939 & $\mathrm{C}^{2} \mathrm{P}$ & 65.8 & 0 & 20.0 & 80.0 \\
\hline \multirow{4}{*}{$\mathrm{N} 64003.90$} & $7 / 2$ & 64001 & $\mathrm{C}^{2} \mathrm{~F}$ & 83.7 & 0 & 1.3 & 98.7 \\
\hline & $3 / 2$ & 65221 & $C \stackrel{3}{2} \mathrm{D}$ & 50.8 & 0.2 & 4.3 & 95.5 \\
\hline & $3 / 2$ & 77750 & $\mathrm{C}_{1}^{2} \mathrm{D}$ & 88.5 & 0.9 & 0.4 & 98.7 \\
\hline & $5 / 2$ & 79860 & $\mathrm{C}_{1}^{2} \mathrm{D}$ & 67.0 & 0.5 & 0.1 & 99.4 \\
\hline
\end{tabular}

Note: $\mathrm{N}$-new energy level.

\section{The Predicted Low Configurations of Pt III}

The spectrum of Pt III is still unknown but, for application to $\mathrm{Pt}$ II, its low energy levels can be predicted by means of the Slater-Condon method. By comparing the lowest energy levels of $5 d^{N}, 5 d^{N-1} 6 s$ and $5 d^{N-2} 6 s^{2}$ in Hf III $(N=2)$ [6], W III $(N=4)$ [7], $\mathrm{Au}$ III $(N=9)$ [8] and $\mathrm{Hg}$ III $(N=10)$ [9], one can reasonably assume that the excitation energies of $5 d^{7} 6 s{ }^{5} \mathrm{~F}_{5}$ and $5 d^{6} 6 s^{25} \mathrm{~F}_{5}$ and $5 d^{6} 6 s^{25} \mathrm{D}_{4}$ levels above the ground level $5 d^{8}{ }^{3} \mathrm{~F}_{4}$ are about 20000 and 60000 $\mathrm{cm}^{-1}$, respectively. All other parameters needed for describing $(5 d+6 s)^{8}$ in Pt III may be obtained from regular trends investigated in second spectra
[3] and in third spectra. The results of this preliminary study are summarized below.

For all $J$-values, the configuration $5 d^{8}$ does not overlap the energy range of the $5 d^{7} 6 s$ and $5 d^{6} 6 s^{2}$ configurations, but this does not prevent configuration mixing. The effect of $5 d^{6} 6 \mathrm{~s}^{2}$ is a constant shift of about $-800 \mathrm{~cm}^{-1}$ for all levels of $5 d^{8}$ except ${ }^{3} \mathrm{P}_{0}$ and ${ }^{1} \mathrm{~S}_{0}$, both shifted by $-1400 \mathrm{~cm}^{-1}$. The effect of the $5 d^{7} 6 s-5 d^{8}$ mixing is more selective and is reported in the last column of Table 3 . These shifts mean that the $5 d^{8}$ parameters would certainly differ if fitted in the approximation of isolated configurations or in mixed groups $(5 d+6 s)^{N}$. The $L S$ names are well defined except for the $J=2$ levels, for which ${ }^{3} \mathrm{P}_{2}$ is nowhere the leading component of 
Table 3. Energy levels of Pt III $5 d^{8}$ predicted in the parametric study of $(5 d+6 s)^{8}$

\begin{tabular}{|c|c|c|c|c|c|c|c|c|c|}
\hline \multirow{2}{*}{$\begin{array}{l}J \\
4\end{array}$} & \multirow{2}{*}{$\begin{array}{r}\begin{array}{c}\text { Energy } \\
\mathrm{cm}^{-1}\end{array} \\
0\end{array}$} & \multirow{2}{*}{$\begin{array}{c}\begin{array}{c}5 d^{8} \text { purity } \\
\%\end{array} \\
98.8\end{array}$} & \multicolumn{2}{|c|}{$\begin{array}{c}\text { First comp. } \\
\%\end{array}$} & \multicolumn{2}{|c|}{ Second comp. } & \multicolumn{2}{|c|}{$\begin{array}{r}\text { Third comp. } \\
\%\end{array}$} & \multirow{2}{*}{$\begin{array}{c}\begin{array}{c}\text { Shift }\left(\mathrm{cm}^{-1}\right) \\
d^{7} s-d^{8}\end{array} \\
-800\end{array}$} \\
\hline & & & ${ }^{3} \mathrm{~F}$ & 94.7 & ${ }^{1} \mathrm{G}$ & 4.0 & & & \\
\hline 2 & 5547 & 94.4 & ${ }^{1} \mathrm{D}$ & 41.1 & ${ }^{3} \mathbf{P}$ & 37.7 & ${ }^{3} \mathrm{~F}$ & 16.3 & -3350 \\
\hline 3 & 9859 & 98.4 & ${ }^{3} \mathrm{~F}$ & 98.4 & $\left({ }^{2} \mathrm{~F}\right)^{3} \mathrm{~F}$ & 1.1 & & & -1000 \\
\hline 2 & 14249 & 93.2 & ${ }^{3} \mathrm{~F}$ & 47.6 & ${ }^{3} \mathbf{P}$ & 40.6 & ${ }^{1} \mathrm{D}$ & 5.1 & -3050 \\
\hline 0 & 15127 & 93.4 & ${ }^{3} \mathrm{P}$ & 81.5 & ${ }^{1} \mathrm{~S}$ & 11.9 & $\left({ }^{2} \mathrm{P}\right)^{3} \mathrm{P}$ & 5.5 & -3800 \\
\hline 1 & 16700 & 89.7 & ${ }^{3} \mathbf{P}$ & 89.7 & $\left({ }^{2} P\right)^{3} P$ & 8.4 & & & -4950 \\
\hline 4 & 21675 & 89.8 & ${ }^{\mathrm{I}} \mathrm{G}$ & 86.0 & $\left({ }^{2} G\right)^{1} G$ & 5.8 & ${ }^{3} \mathbf{F}$ & 3.9 & -2850 \\
\hline 2 & 24760 & 92.5 & ${ }^{1} \mathrm{D}$ & 48.0 & ${ }^{3} \mathrm{~F}$ & 33.9 & ${ }^{3} \mathrm{P}$ & 10.7 & -3200 \\
\hline 0 & 46301 & 60.3 & 's & 58.3 & $\left({ }^{2} \mathrm{P}\right)^{3} \mathrm{P}$ & 28.0 & $\left({ }^{4} \mathrm{P}\right){ }^{3} \mathrm{P}$ & 10.6 & -1350 \\
\hline
\end{tabular}

the eigenfunction. Since the second and third $J=2$ levels have respectively dominant ${ }^{3} \mathrm{~F}_{2}$ and ${ }^{1} \mathrm{D}_{2}$ characters, the lowest $J=2$ level has been given the designation ${ }^{3} \mathrm{P}_{2}$ for identification purposes in the next step of the work.

\section{Interpretation of the Upper Even Configurations}

Nine high even levels were identified by Shenstone [4] as $5 d^{8} 7 s$ and $5 d^{8} 6 d$. One of these levels has now been rejected and the $J$-values of two revised. The three levels of $5 d^{8} 8 s$ and $5 d^{8} 7 d$ have not been confirmed. Thirty-two levels have been found between 101500 and $121700 \mathrm{~cm}^{-1}$. The intensity of their transitions and some relatively large deviations $E_{\text {exp }}-E_{\text {th }}$ in the separate studies of these configurations led us to evaluate their mixing. The 21 integrals needed to describe the levels of $5 d^{8} 7 s+5 d^{8} 6 d$ were reduced to 15 adjustable parameters by means of constraints given in Table 1 . The mixing of the lowest $J=1 / 2$ levels leads to a well-defined value for the interaction parameter $R^{2}(5 d 7 s, 5 d 6 d)$ and the final rms deviation is 79 $\mathrm{cm}^{-1}$. As shown in Table 1 , the values of the parameters $F^{2}(5 d, 5 d)$ and $\alpha$ for $5 d^{8}(6 d+7 s)$ differ significantly from those for $5 d^{7} 6 s^{2}$ and $5 d^{8} 6 s$; however, the parameters are well-defined in the leastsquares fit. We consider this to be an effect of truncation problems discussed in Sec. 3. It seems likely that these inconsistencies would be corrected if all six configurations $(5 d+6 s)^{8} 7 s+(5 d+6 s)^{8} 6 d$ were studied together. This extended parametric study has not been undertaken because $5 d^{6} 6 s^{2} 7 s$, $5 d^{6} 6 s^{2} 6 d$ and $5 d^{7} 6 s 6 d$ are totally unknown and only two levels of $5 d^{7} 6 s 7 s$ are located so far. The predictions of our restricted study might well be unreliable and the theoretical energies of unknown levels have therefore not been reported here.

\section{Odd Levels of Pt II}

The lowest odd levels were attributed to $5 d^{8} 6 p$ by Shenstone [4]. This configuration is also known in other ions of the isoelectronic sequence through Bi VII [9-11]. The approximation of an isolated $5 d^{8} 6 p$ configuration, if valid, has been used for the theoretical study of Au III-Bi VII spectra. It does not hold for Pt II. In second spectra, the overlap of $5 d^{N} 6 p, 5 d^{N-1} 6 s 6 p$ and $5 d^{N-2} 6 s^{2} 6 p$ requires a multiconfigurational treatment. In Hf II, Ta II, W II, $\mathrm{Au}$ II and $\mathrm{Hg}$ II [12], these low odd configurations had been interpreted with rms deviations smaller than $200 \mathrm{~cm}^{-1}$. For unclear reasons, the rms deviation for $\mathrm{Pt}$ II is larger than $500 \mathrm{~cm}^{-1}$ and the designations reported in Table 4 are carefully limited to the lowest levels. Some of them might well be revised with further advances in the parametric interpretation. The $5 d^{7} 6 s 6 p$ configuration starts with the 62820 level, for which we explain the absence of decay to $5 d^{7} 6 s^{2}{ }^{4} F_{9 / 2}$ by the selection rule on the strongly forbidden transition $6 s 6 p{ }^{3} \mathrm{P}_{0}-6 s^{2}{ }^{1} \mathrm{~S}_{0}$. Most of the levels without designation belong to $5 d^{7} 6 s 6 p$ with some admixture of $5 d^{6} 6 s^{2} 6 p$ for the highest energies.

\section{Conclusion}

The strongest unclassified lines of Pt II have been interpreted by extending the early analysis of Shenstone with the help of accurate wavelength measurements and parametric calculations of the main configurations. The number of levels has been brought from 29 to 72 in the even parity and from 71 to 174 in the odd parity. The theoretical study stresses the importance of the $5 d^{8}-5 d^{7}$ 6s interaction and, although somewhat preliminary, the parametric interpretation of the low odd levels indicates that all levels with $J=3 / 2$ through $11 / 2$ below $79000 \mathrm{~cm}^{-1}$ have been found. 
Table 4. Upper even levels of Pt II. The theoretical energies $E_{\text {th }}$ are from the parametric study of $5 d^{8} 6 d+5 d^{8} 7 s$. The core term of $5 d^{8}$ is indicated in parenthesis for $5 d^{8} 6 d$ only

\begin{tabular}{|c|c|c|c|c|c|c|c|}
\hline $\begin{array}{c}E_{\exp } \\
\left(\mathrm{cm}^{-1}\right)\end{array}$ & $J$ & $\begin{array}{c}E_{\text {th }} \\
\left(\mathrm{cm}^{-1}\right)\end{array}$ & Designation & $5 d^{4} 7 s \%$ & $5 d^{8} 6 d \%$ & Leading $L S$ co & omp. \% \\
\hline 95803.363 & $9 / 2$ & 95837 & $\left({ }^{3} F_{4}\right) 7 s_{1 / 2}$ & 99.8 & 0.2 & $7 s^{4} \mathrm{~F}$ & 95 \\
\hline 96614.352 & $7 / 2$ & 96630 & $\left({ }^{3} \mathrm{~F}_{4}\right) 7 s_{1 / 2}$ & 99.9 & 0.1 & $7 s^{2} \mathrm{~F}$ & 66 \\
\hline 101199.085 & $5 / 2$ & 101199 & $\left({ }^{3} \mathrm{P}_{2}\right) 7 s_{1 / 2}$ & 97.6 & 2.4 & $7 s^{2} \mathrm{D}$ & 43 \\
\hline N 101517.59 & $3 / 2$ & 101500 & $\left({ }^{3} \mathrm{P}_{2}\right) 7 s_{1 / 2}$ & 99.5 & 0.5 & $7 s^{2} \mathrm{D}$ & 46 \\
\hline N 104090.70 & $7 / 2$ & 104210 & $\left({ }^{3} F_{4}\right) 6 d_{3 / 2}$ & 0.9 & 99.1 & $6 d\left({ }^{3} \mathrm{~F}\right)^{4} \mathrm{D}$ & 62 \\
\hline$N 104410.05$ & $11 / 2$ & 104405 & $\left({ }^{3} F_{4}\right) 6 d_{3 / 2}$ & 0 & 100. & $6 d\left({ }^{3} \mathrm{~F}\right)^{2} \mathrm{H}$ & 46 \\
\hline J 104636.905 & $9 / 2$ & 104612 & $\left({ }^{3} F_{4}\right) 6 d_{3 / 2}$ & 0.1 & 99.9 & $6 d\left({ }^{3} \mathrm{~F}\right)^{4} \mathrm{~F}$ & 36 \\
\hline 104763.45 & $13 / 2$ & 104698 & $\left({ }^{3} F_{4}\right) 6 d_{5 / 2}$ & 0 & 100. & $6 d\left({ }^{3} \mathrm{~F}\right)^{4} \mathrm{H}$ & 95 \\
\hline N 104930.26 & $3 / 2$ & 105955 & $\left({ }^{3} F_{4}\right) 6 d_{5 / 2}$ & 0.7 & 99.3 & $6 d\left({ }^{3} F\right)^{2} P$ & 58 \\
\hline 105066.347 & $11 / 2$ & 105029 & $\left({ }^{3} F_{4}\right) 6 d_{5 / 2}$ & 0 & 100. & $6 d\left({ }^{3} F\right)^{4} G$ & 72 \\
\hline N 105086.83 & $7 / 2$ & 105046 & $\left({ }^{3} \mathrm{~F}_{4}\right) 6 d_{5 / 2}$ & 0.1 & 99.9 & $6 d\left({ }^{3} F\right)^{2} F$ & 61 \\
\hline 105388.130 & $9 / 2$ & 105413 & $\left({ }^{3} F_{4}\right) 6 d_{5 / 2}$ & 0 & 100. & $6 d\left({ }^{3} F\right)^{2} G$ & 48 \\
\hline N 105794.53 & $7 / 2$ & 105739 & $\left({ }^{3} F_{3}\right) 7 s_{1 / 2}$ & 98.9 & 1.1 & $7 s{ }^{4} \mathrm{~F}$ & 69 \\
\hline N 105962.52 & $5 / 2$ & 105880 & $\left({ }^{3} F_{3}\right) 7 s_{1 / 2}$ & 99.0 & 1.0 & $7 s^{4} \mathrm{~F}$ & 59 \\
\hline J 106434.92 & $5 / 2$ & 106430 & $\left({ }^{3} F_{4}\right) 6 d_{5 / 2}$ & 1.1 & 98.9 & $6 d\left({ }^{3} \mathrm{~F}\right)^{2} \mathrm{D}$ & 39 \\
\hline N 109346.33 & $3 / 2$ & 109412 & $\left({ }^{3} \mathrm{P}_{2}\right) 6 d_{3 / 2}$ & 3.5 & 96.5 & $6 d\left({ }^{t} \mathrm{D}\right)^{2} \mathrm{D}$ & 37 \\
\hline N 109507.99 & $1 / 2$ & 109472 & $\left({ }^{3} \mathrm{P}_{2}\right) 6 d_{3 / 2}$ & 43.8 & 56.2 & $7 s{ }^{4} \mathrm{~F}$ & 32 \\
\hline N 109527.87 & $5 / 2$ & 109446 & $\left({ }^{3} \mathrm{P}_{2}\right) 6 d_{3 / 2}$ & 3.3 & 96.7 & $6 d\left({ }^{1} \mathrm{D}\right)^{2} \mathrm{~F}$ & 37 \\
\hline N 109676.18 & $7 / 2$ & 109676 & $\left({ }^{3} \mathrm{P}_{2}\right) 6 d_{3 / 2}$ & 0.2 & 99.8 & $6 d\left({ }^{1} D\right)^{2} G$ & 24 \\
\hline N 110020.85 & $1 / 2$ & 110077 & $\left({ }^{3} \mathrm{P}_{2}\right) 6 d_{5 / 2}$ & 10.7 & 89.3 & $6 d\left({ }^{1} \mathrm{D}\right)^{2} \mathrm{P}$ & 40 \\
\hline N 110146.80 & $7 / 2$ & 110061 & $\left({ }^{3} \mathrm{P}_{2}\right) 6 d_{5 / 2}$ & 0 & 100. & $6 d\left({ }^{t} \mathrm{D}\right)^{2} \mathrm{~F}$ & 23 \\
\hline N 110158.16 & $5 / 2$ & 110261 & $\left({ }^{3} F_{2}\right) 7 s_{t / 2}$ & 94.6 & 5.6 & $7 s^{4} \mathbf{P}$ & 44 \\
\hline N 110257.49 & $9 / 2$ & 110313 & $\left({ }^{3} \mathrm{P}_{2}\right) 6 d_{5 / 2}$ & 0.1 & 99.9 & $6 d\left({ }^{1} \mathrm{D}\right)^{2} \mathrm{G}$ & 45 \\
\hline N 110258.18 & $3 / 2$ & 110356 & $\left({ }^{3} F_{2}\right) 7 s_{1 / 2}$ & 93.9 & 6.1 & $7 s{ }^{4} \mathrm{~F}$ & 42 \\
\hline N 110408.02 & $3 / 2$ & 110530 & $\left({ }^{3} \mathrm{~F}_{2}\right) 6 d_{s / 2}$ & 2.1 & 97.9 & $6 d\left({ }^{1} \mathrm{D}\right)^{2} \mathrm{P}$ & 39 \\
\hline$N 111162.69$ & $5 / 2$ & 111075 & $\left({ }^{3} \mathrm{P}_{2}\right) 6 d_{5 / 2}$ & 1.6 & 98.4 & $6 d\left({ }^{1} \mathrm{D}\right)^{2} \mathrm{D}$ & 32 \\
\hline N 111371.71 & $1 / 2$ & 111309 & $\left({ }^{3} \mathrm{P}_{0}\right) 7 s_{\mathrm{t} / 2}$ & 45.6 & 54.4 & $7 s{ }^{4} \mathrm{P}$ & 33 \\
\hline N 112433.31 & $3 / 2$ & 112371 & $\left({ }^{3} \mathrm{P}_{1}\right) 7 s_{\mathrm{t} / 2}$ & 90.9 & 9.1 & $7 s^{4} \mathrm{P}$ & 65 \\
\hline N 113119.61 & $1 / 2$ & 113112 & $\left({ }^{3} \mathbf{P}_{1}\right) 7 s_{1 / 2}$ & 93.1 & 6.9 & $7 s^{2} \mathbf{P}$ & 76 \\
\hline N 114127.60 & $9 / 2$ & 114088 & $\left({ }^{3} \mathrm{~F}_{3}\right) 6 d_{3 / 2}$ & 0.1 & 99.9 & $6 d\left({ }^{3} \mathrm{~F}\right)^{4} \mathrm{H}$ & 58 \\
\hline N 114256.30 & $7 / 2$ & 114179 & $\left({ }^{3} \mathrm{~F}_{3}\right) 6 d_{3 / 2}$ & 0 & 100. & $6 d\left({ }^{3} \mathrm{~F}\right)^{4} \mathrm{G}$ & 48 \\
\hline N 114455.05 & $5 / 2$ & 114530 & $\left(\mathrm{~F}_{3}\right) 6 d_{3 / 2}$ & 0.3 & 99.7 & $6 d\left({ }^{3} \mathrm{~F}\right)^{4} \mathrm{D}$ & 40 \\
\hline N 114539.25 & $11 / 2$ & 114549 & $\left({ }^{3} \mathrm{~F}_{3}\right) 6 d_{5 / 2}$ & 0 & 100. & $6 d\left({ }^{3} \mathrm{~F}\right)^{4} \mathrm{H}$ & 63 \\
\hline N 114861.32 & $9 / 2$ & 114823 & $\left({ }^{3} \mathrm{~F}_{3}\right) 6 d_{5 / 2}$ & 0.1 & 99.9 & $6 d\left({ }^{3} F\right)^{4} G$ & 54 \\
\hline N 115060.84 & $5 / 2$ & 115144 & $\left({ }^{3} F_{3}\right) 6 d_{5 / 2}$ & 0.2 & 99.8 & $6 d\left({ }^{3} \mathrm{~F}\right)^{2} \mathrm{D}$ & 31 \\
\hline N 116689.04 & $9 / 2$ & & $5 d^{7} 6 s 7 s$ & & & & \\
\hline N 117340.84 & $9 / 2$ & 117404 & $\left({ }^{1} G_{4}\right) 7 s_{1 / 2}$ & 98.7 & 1.3 & $7 s^{2} \mathrm{G}$ & 94 \\
\hline N 117493.46 & $7 / 2$ & 117437 & $\left({ }^{1} G_{4}\right) 7 s_{1 / 2}$ & 96.6 & 3.4 & $7 s^{2} \mathrm{G}$ & 92 \\
\hline N 119057.05 & $5 / 2$ & a & & & & & \\
\hline N 121651.19 & $9 / 2$ & & $5 d^{7} 6 s 7 s$ & & & & \\
\hline
\end{tabular}

"Undetermined identification; theoretical $J=5 / 2$ levels are calculated at 119011 and $119177 \mathrm{~cm}^{-1}$.

Notes: $\mathrm{N}-$ new energy level.

$\mathrm{J}-$ revised $J$-value. 
Table 5. Odd energy levels of Pt II

\begin{tabular}{|c|c|c|c|c|c|c|c|c|c|}
\hline \multicolumn{2}{|c|}{$E\left(\mathrm{~cm}^{-1}\right)$} & \multirow{2}{*}{$\begin{array}{c}J \\
7 / 2\end{array}$} & \multirow{2}{*}{$\begin{array}{c}\text { Configuration } \\
5 d^{8} 6 p\end{array}$} & \multirow{2}{*}{$\frac{\text { Designation }}{\left({ }^{3} \mathrm{~F}_{4}\right) 6 p_{1 / 2}}$} & \multicolumn{2}{|c|}{$E\left(\mathrm{~cm}^{-1}\right)$} & \multirow{2}{*}{$\frac{J}{11 / 2}$} & \multirow{2}{*}{$\begin{array}{c}\text { Configuration } \\
5 d^{7} 6 s 6 p\end{array}$} & \multirow[t]{2}{*}{ Designation } \\
\hline & 51408.370 & & & & $\mathrm{~N}$ & 83538.53 & & & \\
\hline & 53875.493 & $9 / 2$ & $5 d^{8} 6 p$ & $\left({ }^{3} F_{4}\right) 6 p_{1 / 2}$ & & 84182.633 & $9 / 2$ & & \\
\hline & 56587.934 & $3 / 2$ & $5 d^{8} 6 p$ & $\left({ }^{3} \mathrm{P}_{2}\right) 6 p_{1 / 2}$ & $\mathrm{E}, \mathrm{J}$ & 85700.27 & $9 / 2$ & $5 d^{7} 6 s 6 p$ & \\
\hline & 57018.130 & $5 / 2$ & $5 d^{8} 6 p$ & $\left({ }^{3} P_{2}\right) 6 p_{1 / 2}$ & $\mathrm{~N}$ & 85775.64 & $11 / 2$ & & \\
\hline & 60907.688 & $9 / 2$ & $5 d^{8} 6 p$ & $\left({ }^{3} \mathrm{~F}_{4}\right) 6 p_{3 / 2}$ & $\mathrm{~N}$ & 85826.57 & $7 / 2$ & & \\
\hline & 61058.490 & $11 / 2$ & $5 d^{8} 6 p$ & $\left({ }^{3} F_{4}\right) 6 p_{3 / 2}$ & $\mathrm{~N}$ & 86489.76 & $5 / 2$ & & \\
\hline & 61190.026 & $5 / 2$ & $5 d^{8} 6 p$ & $\left({ }^{3} F_{4}\right) 6 p_{3 / 2}$ & $\mathrm{~N}$ & 87204.35 & $3 / 2$ & & \\
\hline & 61665.485 & $7 / 2$ & $5 d^{8} 6 p$ & $\left({ }^{3} F_{4}\right) 6 p_{3 / 2}$ & $\mathbf{N}$ & 88110.30 & $3 / 2,(5 / 2)$ & & \\
\hline & 62781.658 & $1 / 2$ & $5 d^{8} 6 p$ & $\left({ }^{3} \mathrm{P}_{2}\right) 6 p_{3 / 2}$ & $\mathrm{~N}$ & 88173.46 & $7 / 2$ & & \\
\hline & 62820.489 & $9 / 2$ & $5 d^{7} 6 s 6 p$ & $\left({ }^{4} \mathrm{~F}_{y / 2},{ }^{3} \mathrm{P}_{0}\right)$ & $\mathrm{N}$ & 88589.53 & $7 / 2$ & & \\
\hline & 63738.841 & $7 / 2$ & $5 d^{8} 6 p$ & $\left({ }^{3} F_{3}\right) 6 p_{1 / 2}$ & $\mathrm{~N}$ & 89095.05 & $3 / 2$ & & \\
\hline & 64388.642 & $3 / 2$ & $5 d^{8} 6 p$ & $\left({ }^{3} \mathrm{~F}_{2}\right) 6 p_{1 / 2}$ & & 89607.936 & $5 / 2$ & & \\
\hline & 64757.343 & $5 / 2$ & $5 d^{8} 6 p$ & $\left({ }^{3} \mathrm{~F}_{3}\right) 6 p_{1 / 2}$ & $\mathrm{E}$ & 89863.27 & $9 / 2$ & & \\
\hline \multirow[t]{5}{*}{$\mathrm{N}$} & 65046.23 & $11 / 2$ & $5 d^{7} 6 s 6 p$ & $\left({ }^{4} \mathrm{~F}_{9 / 2},{ }^{3} \mathrm{P}_{1}\right)$ & $\mathrm{N}$ & 90173.25 & $7 / 2,9 / 2$ & & \\
\hline & 65351.069 & $5 / 2$ & $5 d^{8} 6 p$ & $\left({ }^{3} \mathrm{P}_{2}\right) 6 p_{3 / 2}$ & $\mathrm{~N}$ & 90746.64 & $5 / 2$ & & \\
\hline & 65587.115 & $1 / 2$ & $5 d^{8} 6 p$ & $\left({ }^{3} P_{0}\right) 6 p_{1 / 2}$ & $\mathbf{N}$ & 91016.64 & $1 / 2,3 / 2$ & & \\
\hline & 66028.014 & $3 / 2$ & $5 d^{8} 6 p$ & $\left({ }^{3} \mathrm{P}_{2}\right) 6 p_{3 / 2}$ & $\mathrm{~N}$ & 91271.16 & $5 / 2$ & & \\
\hline & 66434.315 & $7 / 2$ & $5 d^{8} 6 p$ & $\left({ }^{3} \mathrm{P}_{2}\right) 6 p_{3 / 2}$ & $\mathbf{N}$ & 91669.95 & $7 / 2$ & & \\
\hline$N$ & 67780.44 & $7 / 2$ & $5 d^{7} 6 s 6 p$ & $\left({ }^{4} \mathrm{~F}_{9 / 2},{ }^{3} \mathrm{P}_{1}\right)$ & $\mathbf{N}$ & 92526.90 & $11 / 2$ & $5 d^{7} 6 s 6 p$ & \\
\hline \multirow[t]{4}{*}{ J } & 69235.665 & $3 / 2$ & $5 d^{8} 6 p$ & $\left({ }^{3} \mathrm{P}_{1}\right) 6 p_{1 / 2}$ & $\mathrm{~N}$ & 92537.08 & $9 / 2$ & & \\
\hline & 69953.317 & $5 / 2$ & $5 d^{8} 6 p$ & $\left({ }^{3} F_{3}\right) 6 p_{3 / 2}$ & $\mathbf{N}$ & 92749.02 & $3 / 2$ & & \\
\hline & 70181.281 & $9 / 2$ & $5 d^{7} 6 s 6 p$ & $\left({ }^{4} \mathrm{~F}_{9 / 2},{ }^{3} \mathrm{P}_{1}\right)$ & $\mathrm{N}$ & 92767.97 & $3 / 2$ & & \\
\hline & 70379.023 & $5 / 2$ & $5 d^{8} 6 p$ & $\left({ }^{3} \mathrm{P}_{1}\right) 6 p_{3 / 2}$ & $\mathrm{~N}$ & 93197.46 & $5 / 2$ & & \\
\hline $\mathbf{N}$ & 71021.13 & $9 / 2$ & $5 d^{8} 6 p$ & $\left({ }^{3} \mathrm{~F}_{3}\right) 6 p_{3 / 2}$ & $\mathbf{N}$ & 93322.18 & $11 / 2$ & $5 d^{7} 6 s 6 p$ & \\
\hline J & 71314.594 & $7 / 2$ & $5 d^{8} 6 p$ & $\left({ }^{3} \mathrm{~F}_{3}\right) 6 p_{3 / 2}$ & & 93336.287 & $7 / 2$ & & \\
\hline \multirow[t]{5}{*}{$\mathrm{N}$} & 71364.68 & $3 / 2$ & $5 d^{8} 6 p$ & $\left({ }^{3} \mathrm{~F}_{3}\right) 6 p_{3 / 2}$ & & 93482.013 & $7 / 2$ & & \\
\hline & 71948.916 & $5 / 2$ & $5 d^{7} 6 s 6 p$ & & $\mathrm{E}$ & 94022.39 & $9 / 2$ & & \\
\hline & 73026.380 & $3 / 2$ & $5 d^{8} 6 p$ & $\left({ }^{3} \mathrm{~F}_{2}\right) 6 p_{3 / 2}$ & $\mathrm{~N}$ & 94271.53 & $5 / 2$ & & \\
\hline & 73431.346 & $9 / 2$ & $5 d^{8} 6 p$ & $\left({ }^{1} G_{4}\right) 6 p_{1 / 2}$ & $\mathbf{N}$ & 94633.25 & $5 / 2$ & & \\
\hline & 73761.739 & $7 / 2$ & & & $\mathbf{N}$ & 94829.73 & $1 / 2,3 / 2$ & & \\
\hline \multirow[t]{2}{*}{ E,J } & 73999.85 & $5 / 2$ & & & $\mathbf{N}$ & 94842.49 & $5 / 2$ & & \\
\hline & 74241.479 & $3 / 2$ & $5 d^{8} 6 p$ & & $\mathbf{N}$ & 95226.00 & $9 / 2$ & & \\
\hline \multirow[t]{5}{*}{$\mathbf{N}$} & 74409.47 & $11 / 2$ & $5 d^{7} 6 s 6 p$ & $\left({ }^{4} F_{9 / 2},{ }^{3} P_{2}\right)$ & $\mathbf{N}$ & 95557.71 & $3 / 2$ & & \\
\hline & 74619.107 & $5 / 2$ & & & $\mathbf{N}$ & 95617.03 & $7 / 2$ & & \\
\hline & 74745.916 & $7 / 2$ & & & $\mathbf{E}$ & 95754.07 & $5 / 2$ & & \\
\hline & 74754.823 & $1 / 2$ & $5 d^{8} 6 p$ & $\left({ }^{3} \mathrm{~F}_{2}\right) 6 p_{3 / 2}$ & $\mathbf{N}$ & 96109.73 & $11 / 2$ & & \\
\hline & 75184.880 & $7 / 2$ & $5 d^{8} 6 p$ & $\left({ }^{1} G_{4}\right) 6 p_{1 / 2}$ & $\mathbf{N}$ & 96131.24 & $9 / 2$ & & \\
\hline \multirow[t]{5}{*}{$\mathbf{N}$} & 75365.84 & $5 / 2$ & & & $\mathrm{~N}$ & 96403.32 & $5 / 2$ & & \\
\hline & 75581.422 & $3 / 2$ & & & $\mathrm{~N}$ & 96443.92 & $1 / 2$ & & \\
\hline & 76461.526 & $5 / 2$ & & & $\mathbf{N}$ & 97183.40 & $3 / 2$ & & \\
\hline & 76610.046 & $3 / 2$ & & & & 97630.600 & $7 / 2$ & & \\
\hline & 77519.724 & $9 / 2$ & & & $\mathrm{~N}$ & 97786.55 & $3 / 2$ & & \\
\hline $\mathrm{N}$ & 77538.25 & $3 / 2$ & & & $\mathrm{~N}$ & 97792.75 & $5 / 2$ & & \\
\hline $\mathbf{N}$ & 77763.58 & $1 / 2$ & & & $\mathbf{J}$ & 98186.971 & $7 / 2$ & & \\
\hline $\mathrm{E}, \mathrm{J}$ & 78043.02 & $7 / 2$ & & & & 98817.744 & $7 / 2$ & & \\
\hline $\mathrm{N}$ & 78254.80 & $5 / 2$ & & & $\mathrm{~N}$ & 99068.74 & $3 / 2$ & & \\
\hline$N$ & 78452.50 & $3 / 2$ & & & & 99209.011 & $5 / 2$ & & \\
\hline & 78906.492 & $9 / 2$ & $5 d^{7} 6 s 6 p$ & $\left({ }^{4} \mathrm{~F}_{9 / 2},{ }^{3} \mathrm{P}_{2}\right)$ & $\mathrm{N}$ & 99471.02 & $1 / 2$ & & \\
\hline$N$ & 79092.09 & $3 / 2$ & & & & 99797.778 & $5 / 2$ & & \\
\hline & 79607.460 & $5 / 2$ & & & $\mathbf{N}$ & 100232.63 & $9 / 2$ & & \\
\hline $\mathbf{N}$ & 79683.41 & $1 / 2$ & & & & 100239.421 & $5 / 2$ & & \\
\hline $\mathrm{N}$ & 80197.33 & $7 / 2$ & $5 d^{8} 6 p$ & $\left({ }^{1} G_{4}\right) 6 p_{3 / 2}$ & & 100611.695 & $3 / 2$ & & \\
\hline & 80858.488 & $5 / 2$ & & & & 100795.666 & $3 / 2$ & & \\
\hline $\mathbf{N}$ & 81083.95 & $9 / 2$ & $5 d^{8} 6 p$ & $\left({ }^{1} G_{4}\right) 6 p_{3 / 2}$ & & 100903.454 & $7 / 2$ & & \\
\hline $\mathrm{E}$ & 81897.71 & $7 / 2$ & & & $\mathbf{N}$ & 101113.06 & $1 / 2$ & & \\
\hline $\mathrm{E}, \mathrm{J}$ & 82535.79 & $5 / 2$ & & & & 101341.867 & $7 / 2$ & & \\
\hline $\mathbf{N}$ & 82692.28 & $9 / 2$ & $5 d^{7} 6 s 6 p$ & & $\mathbf{N}$ & 101394.01 & $1 / 2$ & & \\
\hline $\mathrm{E}$ & 82824.00 & $3 / 2$ & & & & 101397.850 & $5 / 2$ & & \\
\hline E,J & 82972.72 & $3 / 2$ & & & $\mathrm{~J}$ & 101549.10 & $9 / 2$ & & \\
\hline & 83352.251 & $7 / 2$ & & & & 101618.459 & $11 / 2$ & & \\
\hline
\end{tabular}


Table 5. Odd energy levels of Pt II - Continued

\begin{tabular}{|c|c|c|c|c|c|c|c|c|c|}
\hline \multicolumn{2}{|r|}{$E\left(\mathrm{~cm}^{-1}\right)$} & \multirow{2}{*}{$\frac{J}{S / 2}$} & \multirow[t]{2}{*}{ Configuration } & \multirow[t]{2}{*}{ Designation } & \multicolumn{2}{|c|}{$E\left(\mathrm{~cm}^{-1}\right)$} & \multirow{2}{*}{$\begin{array}{c}J \\
9 / 2\end{array}$} & \multirow[t]{2}{*}{ Configuration } & \multirow[t]{2}{*}{ Designation } \\
\hline & 101916.930 & & & & $\mathbf{N}$ & 107386.26 & & & \\
\hline & 102086.034 & $9 / 2$ & & & $\mathbf{N}$ & 107588.13 & $3 / 2$ & & \\
\hline & 102414.857 & $5 / 2$ & & & $\mathbf{N}$ & 108037.26 & $7 / 2$ & & \\
\hline $\mathbf{N}$ & 102520.80 & $7 / 2$ & & & $\mathbf{N}$ & 108038.05 & $3 / 2$ & & \\
\hline $\mathbf{N}$ & 102613.05 & $11 / 2$ & & & $\mathbf{N}$ & 108155.51 & $3 / 2$ & & \\
\hline $\mathbf{N}$ & 102678.30 & $3 / 2$ & & & $\mathbf{N}$ & 108322.40 & $7 / 2$ & & \\
\hline $\mathbf{N}$ & 102872.20 & $7 / 2$ & & & $\mathbf{N}$ & 108639.24 & $5 / 2$ & & \\
\hline $\mathbf{N}$ & 103060.55 & $9 / 2$ & & & $\mathbf{N}$ & 108672.51 & $1 / 2,3 / 2$ & & \\
\hline \multirow[t]{3}{*}{$\mathbf{N}$} & 103421.16 & $3 / 2$ & & & $\mathbf{N}$ & 108727.50 & $7 / 2$ & & \\
\hline & 103463.310 & $5 / 2$ & & & $\mathbf{N}$ & 108802.20 & $7 / 2,9 / 2$ & & \\
\hline & 103517.132 & $7 / 2$ & & & $\mathbf{N}$ & 109307.89 & $7 / 2$ & & \\
\hline $\mathbf{N}$ & 103637.26 & $1 / 2,3 / 2$ & & & $\mathbf{N}$ & 109528.23 & $3 / 2,5 / 2$ & & \\
\hline $\mathbf{N}$ & 104092.10 & $3 / 2,5 / 2$ & & & $\mathbf{N}$ & 109733.10 & $7 / 2$ & & \\
\hline $\mathbf{N}$ & 104158.64 & $5 / 2$ & & & $\mathbf{N}$ & 109753.67 & $5 / 2$ & & \\
\hline $\mathbf{N}$ & 104548.13 & $3 / 2$ & & & $\mathbf{N}$ & 110066.71 & $9 / 2$ & & \\
\hline $\mathbf{N}$ & 104625.27 & $9 / 2$ & & & $\mathbf{N}$ & 110085.70 & $3 / 2$ & & \\
\hline $\mathbf{N}$ & 104831.58 & $9 / 2$ & & & $\mathbf{N}$ & 110196.40 & $5 / 2$ & & \\
\hline $\mathbf{N}$ & 105018.17 & $3 / 2$ & & & $\mathbf{N}$ & 110202.39 & $7 / 2$ & & \\
\hline $\mathbf{N}$ & 105042.36 & $5 / 2$ & & & $\mathbf{N}$ & 110609.08 & $9 / 2$ & & \\
\hline $\mathbf{N}$ & 105554.33 & $7 / 2$ & & & $\mathbf{N}$ & 110638.00 & $5 / 2$ & & \\
\hline $\mathbf{N}$ & 105597.33 & $5 / 2$ & & & $\mathbf{N}$ & 110684.45 & $3 / 2$ & & \\
\hline $\mathbf{N}$ & 105726.12 & $1 / 2$ & & & $\mathbf{N}$ & 110762.77 & $7 / 2,9 / 2$ & & \\
\hline $\mathbf{N}$ & 105896.50 & $3 / 2$ & & & $\mathbf{N}$ & 111320.57 & $5 / 2$ & & \\
\hline $\mathbf{N}$ & 106229.90 & $7 / 2$ & & & $\mathbf{N}$ & 111354.67 & $7 / 2$ & & \\
\hline $\mathbf{N}$ & 106852.84 & $3 / 2$ & & & $\mathbf{N}$ & 111716.41 & $7 / 2,9 / 2$ & & \\
\hline $\mathbf{N}$ & 106995.20 & $9 / 2$ & & & $\mathbf{N}$ & 112247.69 & $9 / 2$ & & \\
\hline $\mathbf{N}$ & 106996.55 & $7 / 2$ & & & $\mathbf{N}$ & 113785.71 & $3 / 2,(1 / 2)$ & & \\
\hline $\mathbf{N}$ & 107191.45 & $3 / 2,5 / 2$ & & & $\mathbf{N}$ & 114880.48 & $5 / 2$ & & \\
\hline
\end{tabular}

a These $J_{-j}$ characters are equally shared by the levels 61190 and 64757 .

Notes: $\mathbf{N}-$ new energy level.

$\mathrm{J}$-revised $J$-value.

E-revised energy value.

\section{Acknowledgments}

We are grateful to Dr. J. Reader and his coworkers for sending us several line lists of platinum as soon as they were completed. The theoretical calculations have been performed by means of the chain of programs written and maintained at Laboratoire Aimé Cotton by $\mathrm{A}$. Bachelier and J. Sinzelle.

\section{References}

[1] J. E. Sansonetti, J. Reader, C. J. Sansonetti, and N. J. Acquista, J. Res. Natl. Inst. Stand. Technol. 97, 1 (1992).

[2] J. Reader, N. Acquista, C. J. Sansonetti, and R. Engleman, Jr., J. Opt. Soc. Am. B5, 2106 (1988).

[3] J.-F. Wyart, Optica Pura Appl. 10, 177 (1977).

[4] A. G. Shenstone, Philos. Trans. R. Soc. London, Ser. A 237, 453 (1983).

[5] B. R. Judd, J. E. Hansen, and A. J. J. Raassen, J. Phys. B: Atom. Molec. Phys. 15, 1457 (1982).
[6] P. F. A. Klinkenberg, Th. A. M. van Kleef, and P. E. Noorman, Physica 27, 151 (1961).

[7] L. Iglesias, M. I. Cabeza, F. R. Rico, O. Garcia-Riquelme, and V. Kaufman, J. Res. Natl. Inst. Stand. Technol. 94, 221 (1989).

[8] L. Iglesias, J. Res. NatI. Bur. Stand. (U.S.) 64 A, 481 (1960); 70 A, 465 (1966).

[9] C. E. Moore, Atomic Energy Levels, Natl. Bur. Stand. (U.S.) Circ. 467, (1959).

[10] Y. N. Joshi, A. J. J. Raassen, and B. Arcimowicz, J. Opt. Soc. Am. B 6, 534 (1989).

[11] A. J. J. Raassen, A. A. van der Valk, Y. N. Joshi, L. I. Podobedova, and A. N. Ryabtsev, J. Phys. B: Atom. Molec. Phys. 22, 809 (1989).

[12] J.-F. Wyart and J. Blaise, Physica Scripta 42, 209 (1990).

About the authors: J. Blaise and J.-F. Wyart are both "Directeur de recherche" at the Centre National de la Recherche Scientifique in Paris. J. Blaise is an Emeritus Fellow of the Optical Society of America and received the W. F. Meggers Award in 1975. J.-F. Wyart was a guest worker at the National Bureau of Standards in 1980. 